\title{
FORECASTING THE NUMBER OF PATIENTS DISEASES USING BACKPROPAGATION
}

\author{
Aeri Rachmad, Devie Rosa Anamisa \\ Faculty of Engineering, University of Trunojoyo Madura, Indonesia \\ Email: aery_r@yahoo.com
}

\begin{abstract}
Forecasting with various types of disease is important for health centers, because it can be used to help the health center management in conducting strategic planning and decision making. Health Care Center Torjun in Indonesia has made estimationabout the number of patients with various types of diseases, such as Acute Respiratory Infections(ISPA), RA (Rheumatoid Arthritis), diarrhea, HT (Hypertension), Skin Allergies, Conjunctivitis, Asthma, Febrile, TB(Tuberculosis) Lung, scabies, Gastritis, typus and scarlet fever with reports the number of patients with certain diseases in the coming period and prepare the necessary needs both medical services and as well as drugs for use later. In this study, Artificial Neural Network (ANN) is one model that is used to identify patterns of images of people with various kinds of diseases. Backpropagation is one of the popular models of Neural Networkwhich is used for forecasting, prediction, and decision makers based on the input of data entry that has been studied in advance. The resultsis HT $(0.35 \%)$ with parameters for forecasting system using Neural Network Backpropagation is the best of the trial results that shows disease HT which are obtained from the experiments. They predict the number of patients with a disease that needs to be watched for in the coming period and prepare all the needs of both medical and medication needed to handle the number of people with the disease.
\end{abstract}

Keywords: Forecasting, Health Care Center, Diseases, Backpropagation

\section{INTRODUCTION}

Forecasting is a process to predict the needs of the future which includes the need to measure the quantity, quality, time and location needed in order to meet the demand for goods and services. Forecasting demand is the level of demand for the products that are expected to be realized for a certain period in the future. Basically forecasting approaches can be classified into two approaches: qualitative approach and quantitative approach (Memmedli, 2012)

There are some solutions that are needed such as a good quality of initial data and in sufficient quantity, because principally, the method of prediction is to identify the pattern of data which is based on the existing data, so mathematically predicted results will be relatively accurate (Chang, 2012). One technique that can be considered in pattern recognition neural network for prediction is a clone. Neural Network Backpropagation is the development of least mean square algorithm that can be used to train the network with multiple layers which is based on steepest descent algorithm approach and uses its performance index as the mean square error (Kara, 2011).

The outcome of a forecasting considers that forecastingmust contain errors, meaning that forecasters could only reduce the uncertainty that will happen, but it can not eliminate this uncertainty. Forecasting should provide information about how large the size of the erroris, meaning that forecasting certainly contains errors.It is important for forecasters to inform how large the errors that may occur, or even short-term forecasting is more accurate than long-term forecasting (Santoso,2009).

Along with the development of technology, there are more and more emerging methods that can be used for forecasting such as the classic method and fuzzy time series. Time series methods for modeling and forecasting monthly number of patients at a health clinic which has produced levels of forecasting accuracy of the existing methods are not good enough. They can only predict seasonal problem, but they failed to foresee problems with linguistic values. Moreover the model also requires a number great historical data to generate accurate forecasting results (Setiyoutami, 2012).

In a previous study, several approaches were made to obtain the results of the best forecasting such as Artificial Neural Network, Ensemble Neural Network, Backpropagation Networks, Radial Basis Function Network, General Regression Neural Network, Genetic Algorithm, Multilayer Perceptron, clustering Fuzzy, etc. To determine the daily weather forecast, it can use back propagation technique with 28 input parameters to foresee some parameters such as temperature, rainfall, humidity, clouds, and weather days (Meera 2015). Instead of predicting the weather phenomenon through traditional ways such as the estimated amount of rainfall, barometric pressure, wind speed, temperature etc, we can use a neural network. Back Propagation neural network has high accuracy and efficiency to predict the weather (Pooja 2014; Meera 2015).

By considering the results of the above studies, this research uses back propagation neural network to predict the number of patients with various types of 13 diseases at the health center. The predictive model is used to estimate the number of patients with a disease that needs to be watched in the coming period.

\section{METHODOLOGY}

Neural networks are information processing systems that have certain characteristics by adapting performance of biological neural network(Deepthi 2015; 
Eka, 2014;Santhosh 2010). An information processing systems is interconnected in a network that work together to produce an output. The output of a neural network has the size on the relationship of each individual node in the network operates. Information processing in neural network is often implemented in parallel and not sequentially. Therefore, the members of neurons which have the same size work together to carry out its function. It is unique from a function of the overall neural network that can still be carried out even if the majority of the neurons are not functioning. That is because the artificial neural networks are very tolerant to faults or failures (Meera 2015). Backpropagation Neural Network may be the reason behind the popularity of artificial intelligence. Because this metode does not restrict the types of algorithms which are used in the learning process. This method spread enthusiasm by the developers of artificial intelligence though it is still much controversy whether the model can be applied as the learning steps of the brain or not. Partly because the mechanism for reversing the signal or error, it can not be accepted. Moreover, because there is no reasonable source for learning signal or target output.Backpropagation uses a multilayer architecture with supervised training methods training. Behind Propagation models, they have a couple of units that exists in one or more hidden layers. Behind Propagation model architecture with $\mathrm{n}$ inputs plus a bias, a hidden layer consisting of $\mathrm{p}$ units plus a bias, and $\mathrm{m}$ pieces of unit output, as shown in Figure 1.

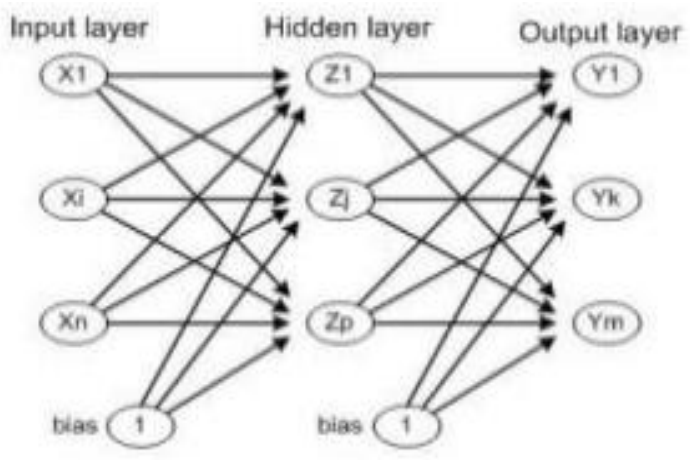

Figurer-1. Backpropagation architecture (MePooja, 2014;

Arti 2012)

ANN consists of many elements called neurons, unit, cell or science node connected to one another via the synaptic link / communication links that have weight-related (Kadar 2011). Weights represent information to be used by the network to resolve the issue. They can be applied to various types of problems, for example for pattern classification, making the mapping of input patterns, grouping with many constraints. Each neuron has an internal state called activity level, where the function of the input has been received. Neurons transmit a signal at a given moment; can be seen in Figure 2.

In the training, ANN Backpropagation initialized the weights to determine the numbers of learning $(\alpha)$, determine the value of the error tolerance or threshold values (when using a threshold value as a condition to stop) or set maximum iterations (when using many iterations as the stop condition), then performed step next-step for the stop condition is worth FALSE.

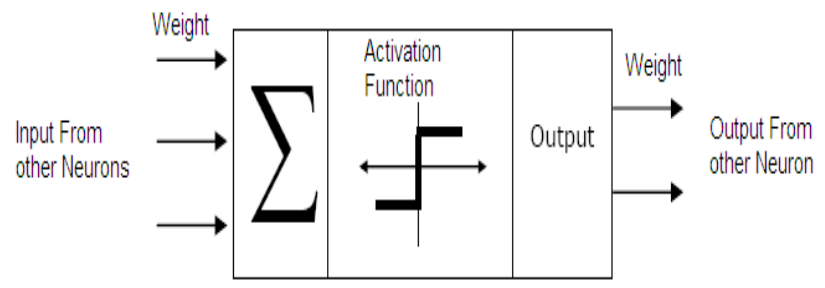

Figure-2. Neuron structure

Every unit of output $\left(\mathrm{y}_{\mathrm{k}}, \mathrm{k}=1,2,3, \ldots, \mathrm{m}\right)$ receives the target pattern associated with the input pattern learning arithmetic error information and then calculate the correction weights (which will be used to improve the value $\mathrm{w}_{\mathrm{jk}}$ ), as in equation 2 and compute their bias correction (which will be used to improve the value $\mathrm{w}_{0 \mathrm{k}}$ ), as in equation 1 (Noviani,2010).

$$
\begin{aligned}
& \delta_{k}=\left(t_{k}-y_{k}\right) f^{\prime}\left(y_{-} i n_{k}\right) \\
& \Delta w_{j k}=\alpha \delta_{k} z_{j} \\
& \Delta w_{0 k}=\alpha \delta_{k}
\end{aligned}
$$

Backpropagation ensures the completion of the minimum mean square error during the learning rate which is not too big. The drawback is when the learning rate is small, then the achievement of convergentvalue is slow, while if the learning rate is large, the achievement of rapid convergence gives a good value, but there is a danger that it can lead to global oscillations when the minimum value is not reached. To overcome this, it used backpropagation variations, such as momentum, where it works with the aim to smooth oscillations that may occur. Filter momentum will be added to the equation weight matrix and bias. Variable Learning Rateworks by trying to raise the learning rate when it encounters a flat surface and then lowering the learning rate in the event of an increase in slope.

\section{RESULTS}

The system of forecasting the number of people with the disease at the health center with Backpropagation ANN method has the design development as in Figure 3.Model neural network with back propagation algorithm has three layers with an input layer consists of several unit cells $\left(\mathrm{x}_{1}-\mathrm{x}_{\mathrm{n}}\right)$ which contains the number of patients disease defined earlier. It can determine the number of people with the disease further, one hidden layer with the number of unit cells is determined at random $\left(\mathrm{y}_{1}-\mathrm{y}_{\mathrm{n}}\right)$, and one layer of output is amounted to one unit cell ( $\mathrm{z}$ ) as the target of the number of patients with disease outcome prediction next period 


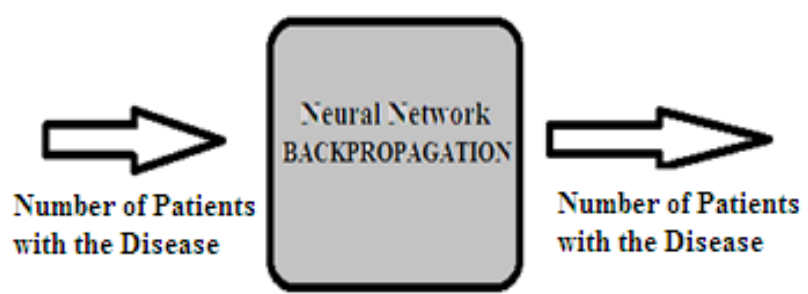

Figure-3. Propose Model for number of patients

$$
X_{i}=y\left(X_{\max }-X_{\min }\right)+X_{\min }
$$

where:

$$
\begin{aligned}
& X_{i}=\text { number of patients with the disease } \\
& Y=\text { the output network } \\
& X_{\min }=\text { Data with a minimum value } \\
& X_{\max }=\text { Data with a maximum value }
\end{aligned}
$$

Table-1. The best parameter configuration

\begin{tabular}{|l|c|c|c|c|c|c|c|}
\hline \multirow{2}{*}{ Disease Name } & \multicolumn{4}{c|}{ Parameter } & \multirow{2}{*}{ MSE } & \multirow{2}{*}{$\begin{array}{c}\text { MAPE } \\
(\%)\end{array}$} \\
\cline { 2 - 7 } & Hidden & Epoh & Mom & Lrate & Error & & 0.24 \\
\hline Acute Respiratory Infections(TSPA) & 2 & 75 & 0.05 & 0.008 & 0.001 & 1567 & 0.001 \\
\hline Rheumatoid Arthritis (RA) & 10 & 50 & 0.02 & 0.008 & 0.001 & 5519 & 32.65 \\
\hline Diarrhea & 4 & 50 & 0.02 & 0.008 & 0.000001 & 295.4 & 4.68 \\
\hline Hypertension (HT) & 2 & 50 & 0.02 & 0.008 & 0.000001 & 57.94 & 0.35 \\
\hline Skin Allergies & 4 & 50 & 0.02 & 0.008 & 0.0001 & 67.63 & 3.91 \\
\hline Conjunctivitis & 2 & 75 & 0.02 & 0.008 & 0.001 & 401.3 & 3.57 \\
\hline Asthma & 2 & 1000 & 0.02 & 0.008 & 0.001 & 289.1 & 15.86 \\
\hline Febrile & 6 & 50 & 0.02 & 0.008 & 0.001 & 422.8 & 9.78 \\
\hline Tuberculosis (TB). Lung & 2 & 50 & 0.05 & 0.008 & 0.001 & 16.76 & 3.14 \\
\hline Scabies & 10 & 1000 & 0.02 & 0.008 & 0.00001 & 0.001 & 0 \\
\hline Gastritis & 6 & 50 & 0.02 & 0.5 & 0.001 & 0.01 & 0 \\
\hline Typus & 8 & 50 & 0.02 & 0.008 & 0.001 & 11.95 & 13.03 \\
\hline Scarlet Fever & 2 & 100 & 0.02 & 0.008 & 0.001 & 1.05 & 0.57 \\
\hline
\end{tabular}

The training process of data patterns is normalized first so that the system can determine the weights that can map between data input with the desired output target data. Normalization is done by dividing the value of data with the value of the data range (maximum data value-minimum data value). Input data normalization is aimed to adjust the value range of data with activation function in the system backpropagation. This means that the value of the square of the input should be in the range of 0 to 1 . Thus, input range that qualifies is the input data values from 0 to 1 or from -1 to 1 . Therefore, the resulting output will be in the range of 0 to 1 , such as in equation 2 (Frank 1997).

$$
f(x)=\frac{X_{i}-X_{\min }}{X_{\max }-X_{\min }}
$$

where:

$$
\begin{aligned}
& \mathrm{X}_{\mathrm{i}} \quad \text { = Data to } \mathrm{i} \\
& \mathrm{X}_{\min } \quad=\text { Data with a minimum value } \\
& \mathrm{X}_{\max } \quad=\text { Data with a maximum value }
\end{aligned}
$$

In the testing process, the output is produced by the network ranges between 0 and 1 so we need denormalization to convert the output result of network back into the normal number of people with the disease, as in Equation 3 (Noviani,2010). After that, it will be a comparison between the actual data with the data of the prediction, so the error can be calculated. calculate the value of the error of the calculation process by the system to the initial value in accordance with the original data using the mean square error (MSE) because MSE is more sensitive to the value of the error. MSE does not have the magnitude of the value. A system is said to have good performance when the MSE value is close to zero (MSE $\approx 0$ ) or can be said to be an error in the system. MSE is calculated by summing the squares of all the forecasting error in each period and dividing by the number of forecast period (Upadhyay 2011).

The trial conducted Backpropagation ANN forecasting model to determine the accuracy of the system in the process of disease predicted number. There are 13 types of disease testing process based on the parameters that are used with real data scenarios comparison with predicted results. Test results of each disease prediction produce the best parameters of different configurations, such as in Table 1.

Each disease produces MSE and MAPE with the best parameter configuration. Configuration parameters generate value system accuracy,which is small.It shows that the difference between the data which predicts results with real data is not large, ascan be seen in Table 2. While the accuracy of the predictions for each disease can be seen in Table 3. 
Table-2. Accuracy with real data and prediction

\begin{tabular}{|l|c|c|c|c|c|c|c|c|c|c|}
\hline \multirow{3}{*}{ Disease Name } & \multicolumn{9}{|c|}{2015} \\
\cline { 2 - 14 } & \multicolumn{2}{|c|}{ January } & \multicolumn{2}{|c|}{ February } & \multicolumn{2}{c|}{ March } & \multicolumn{3}{c|}{ April } & \multicolumn{3}{c|}{ May } \\
\cline { 2 - 13 } & Real & Prediction & Real & Prediction & Real & Prediction & Real & Prediction & Real & Prediction \\
\hline Acute Respiratory Infections(ISPA) & 29 & 208 & 201 & 201 & 209 & 207 & 200 & 198 & 199 & 195 \\
\hline Rheumatoid Arthritis (RA) & 179 & 211 & 180 & 210 & 189 & 213 & 201 & 220 & 187 & 217 \\
\hline Diarrhea & 49 & 45 & 57 & 61 & 61 & 57 & 65 & 60 & 65 & 60 \\
\hline Hypertension (HT) & 30 & 29 & 30 & 31 & 31 & 30 & 36 & 35 & 25 & 24 \\
\hline Skin Allergies & 30 & 25 & 31 & 28 & 37 & 35 & 27 & 30 & 39 & 35 \\
\hline Conjunctivitis & 38 & 35 & 39 & 35 & 41 & 36 & 42 & 36 & 33 & 35 \\
\hline Asthma & 34 & 26 & 32 & 24 & 37 & 25 & 39 & 25 & 32 & 26 \\
\hline Febrile & 33 & 29 & 26 & 31 & 24 & 30 & 36 & 31 & 40 & 32 \\
\hline Tuberculosis (TB). Lung & 8 & 6 & 17 & 16 & 14 & 15 & 18 & 16 & 19 & 16 \\
\hline Scabies & 7 & 7 & 8 & 9 & 8 & 8 & 9 & 8 & 10 & 8 \\
\hline Gastritis & 2 & 3 & 1 & 2 & 1 & 1 & 1 & 2 & 1 & 2 \\
\hline Typus & 6 & 1 & 6 & 2 & 2 & 1 & 5 & 1 & 4 & 1 \\
\hline Scarlet Fever & 2 & 2 & 0 & 1 & 0 & 2 & 1 & 2 & 0 & 2 \\
\hline
\end{tabular}

Tabel-3.Prediction accuracy each disease

\begin{tabular}{|c|c|c|c|c|c|c|c|c|c|c|c|c|c|}
\hline $\begin{array}{l}\text { Disease } \\
\text { Name }\end{array}$ & $\begin{array}{c}\text { Acute } \\
\text { Respiratory } \\
\text { Infections (ISPA) }\end{array}$ & $\begin{array}{c}\text { Rheumatoid } \\
\text { Arthritis } \\
\text { (RA) }\end{array}$ & Diarrhea & $\begin{array}{l}\text { Hypertension } \\
\text { (HT) }\end{array}$ & $\begin{array}{c}\text { Skin } \\
\text { Allergies }\end{array}$ & Conjunctivitis & Asthma & Febrile & $\begin{array}{l}\text { Tuberculosis } \\
\text { (TB). Lung }\end{array}$ & Skabies & Gastritis & Typus & $\begin{array}{l}\text { Scarlet } \\
\text { Fever }\end{array}$ \\
\hline $\begin{array}{c}\text { MAPE } \\
(\%)\end{array}$ & 0.24 & 32.65 & 4.68 & 0.35 & 3.91 & 3.57 & 15.86 & 9.78 & 3.14 & 0 & 0 & 13.03 & 0.57 \\
\hline
\end{tabular}
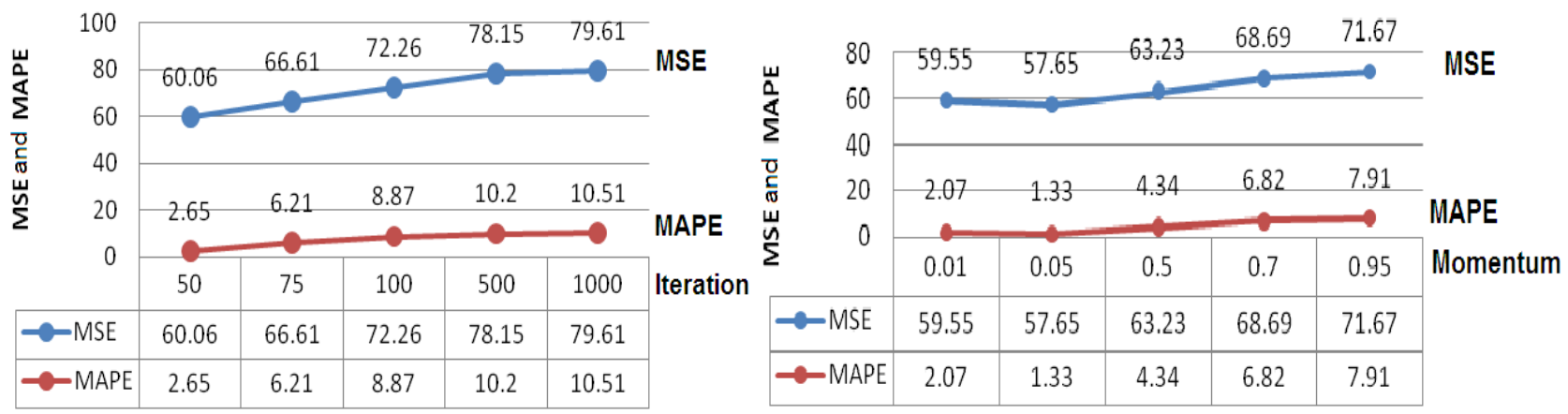

Figure-4. Forecasting evaluation results with iteration and momentum

\section{DISCUSSION}

To evaluate the results of forecasting in this research, it used MAPE. MAPE is the percentage difference between the values of the error of the original data with the results of forecasting. MAPE measures the absolute error as a percentage instead of each period rather than the average absolute error in the number of periods of actual data. It can avoid the problems in the interpretation of measurement accuracy relative to the magnitude of the actual value and the predicted value. From 50 to 1000 iterations MSE and MAPE proportional same for any changes momentum, where the smaller iterations or momentum, the value of MSE and MAPE are getting smaller and vice versa, it is shown in Figure 4. When the comparison of accuracy and the learning rate changes, the smaller the value of learning the higher the rate the accuracy or otherwise. And the value of learning rate 0.008 is resulted in the MSE and MAPE smallest,as shown in Figure 5).

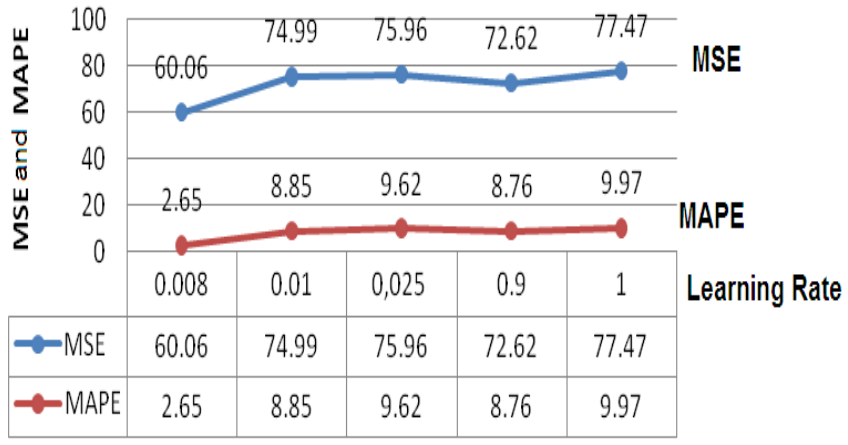

Figure-5. Forecasting evaluation results with learning rate shown in Figure 5).

Artificial neural network architecture consists of one input layer, one hidden layer, and one output layer. The number of input and output node in accordance with the number of lags is obtained from the autocorrelation, while the number of hidden layer is obtained from twice 
the number of input layer nodes. The condition of the system stops if the system generated error value is less than or equal to the value specified error tolerances or the number of iterations performed has reached the maximum number of iterations specified. From some of the experiments that have been done, it can be concluded that the predicted number of patients using the model ANN Backpropagation disease depends on several parameters. In addition to the number of hidden layer, there are other parameters that need to be regulated, namely learning rate and momentum. Learning rate affects the speed and the accuracy of ANN in the learning process. The greater the learning rate, the faster ANN learns but the results are less accurate. The smaller the learning rate, the slower ANN studies but the results are more accurate (Tanu 2015). Momentum effects on the changes in the chart error. The larger the value of momentum, the shape of the curve is getting steeper. The smaller the value of momentum, then the error is getting sloping curve shape. The parameters are chosen to be the best parameter because it produces the number of iterations that has a value reasonably good accuracy error during the system test that is equal to $0.35 \%$.

\section{CONCLUSION}

Configuration parameters for forecasting system using Neural Network Backpropagation is the best of the trial results that shows disease HT which are obtained from the experiments yield learning rate configuration at $0.008,0.02$ momentum, 0.000001 error tolerance, a maximum of 50 iterations and the number of hidden layer 2 as well as a percentage value error $0.35 \%$. At scabies disease, the best parameters were 0.008 learning rate, momentum $0.02,0.00001$ of error tolerance, 1000 maximum iteration, 10 hidden layers, and 0.001 MSE. This shows that the neural network Backpropagation method is suitable to be used for prediction of the number of people with the disease. The smaller the value of learning rate, the better the accuracy of the system. Momentum affects the changes in the chart error. The larger the value of the momentum, the steeper the shape of the error curve. On the opposite, the smaller the value of the momentum, then the flatter the shape of the error curve.

\section{REFERENCES}

Arti R. Naik, Prof. S.K.Pathan. 2012. Weather Classification And Forecasting Using Back Propagation Feed-Forward Neural Network. International Journal of Scientific and Research Publications. 2(12): 1 - 3.

Chang, P. C., Wang, D. d., \& Zhou, C. 1. 2012. A Novel Model By Evolving Partially Connected Neural Network For Stock Price. Expert Systems with Applications. 39: 611-620.

Deepthi.I.Gopinath, G.S. Dwarakish. 2015. Wave Prediction Using Neural Networks At New Mangalore Port Along West Coast Of India, International Conference
On Water Resources. Coastal And Ocean Engineering. Aquatic Procedia. $4: 143-150$.

Eka Malasari R, 2014. Stock Price Predictions Were Considering External Factors Using Artificial Neural Network, Scientific Journals NERO. 1(2): 5 - 11.

Frank M. Thiesing and Oliver Vornberger. 1997. Sales Forecasting Using Neural Networks. IEEE Proceeding ICNN'97 Houston - Texas. 4: $2125-2128$.

Kara, Y., Boyacioglu, M. A., \& Baykan, O. K. 2011. Predicting Direction Of Stock Price Index Movement Using Artificial Neural Networks And Support Vector Machines: The Sample Of The Istanbul Stock Exchange. Expert Systems with Applications 38: 5311-5319.

K. G. Upadhyay, A. K. Choudhary, M. M. Tripathi. 2011. Short-Term Wind Speed Forecasting Using Feed-Forward Back-Propagation Neural Network. International Journal of Engineering, Science and Technology. 3(5): 107-112.

Meera Narvekar, Priyanca Fargose. 2015. Daily Weather Forecasting Using Artificial Neural Network. International Journal of Computer Applications. 121(22): 9- 13.

Memmedli, M., \& Ozdemir, O. 2012. An Empirical Study of Fuzy Aproach with Artificial Neural Network Models. International Journal of Mathematical Models and Methods in Aplied Sciences. 1(4): 321-326.

Noviani, IR. 2010. Decision Support System Housing Sales Price Determination Method Using Backpropagation Neural Network. Thesis: University of Trunojoyo Madura.

Santoso, S. 2009. Business Forecasting: Methods of Business Forecasting Present with MINITAB and SPSS. Jakarta: PT. Elex Media Komputindo.

Setiyoutami, A. 2012. Prediction Poli Surgical Patient Visits in Surabaya Oncology Hospital Fuzy Using Time Series. Thesis: Teknologi Sepuluh Nopember Institute of Surabaya.

S. Santhosh Baboo and I.Kadar Shereef. 2010. An Efficient Weather Forecasting System Using Artificial Neural Network. International Journal Of Environmental Science And Development. 1( 4): 136-142.

Pooja Malik, Prof. Saranjeet Singh, Binni Arora. 2014. An Effective Weather Forecasting Using Neural Network. International Journal of Emerging Engineering Research and Technology. 2( 2 ): 209 - 212.

Tanu Verma, Dr R.K. Srivastava. 2015. Artificial Neural Networks Based Heart Disease Predictive Approach. International Journal of Application or Innovation in Engineering \& Management. 4(3): 029-032. 